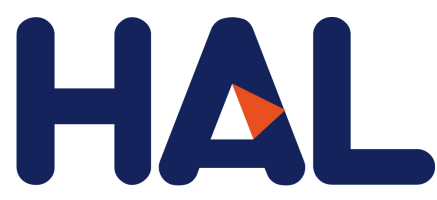

archives-ouvertes

\title{
Environmentally Mediated Social Dilemmas
}

Sylvie Estrela, Eric Libby, Jeremy van Cleve, Florence Débarre, Maxime

Deforet, William Harcombe, Jorge Peña, Sam Brown, Michael Hochberg

\section{To cite this version:}

Sylvie Estrela, Eric Libby, Jeremy van Cleve, Florence Débarre, Maxime Deforet, et al.. Environmentally Mediated Social Dilemmas. Trends in Ecology and Evolution, Elsevier, 2018. hal-01941198

\section{HAL Id: hal-01941198 \\ https://hal.sorbonne-universite.fr/hal-01941198}

Submitted on 30 Nov 2018

HAL is a multi-disciplinary open access archive for the deposit and dissemination of scientific research documents, whether they are published or not. The documents may come from teaching and research institutions in France or abroad, or from public or private research centers.
L'archive ouverte pluridisciplinaire HAL, est destinée au dépôt et à la diffusion de documents scientifiques de niveau recherche, publiés ou non, émanant des établissements d'enseignement et de recherche français ou étrangers, des laboratoires publics ou privés. 


\section{Environmentally mediated social dilemmas}

Sylvie Estrela ${ }^{1,2, * \#, ~ E r i c ~ L i b b y ~}{ }^{3,4, *}$, Jeremy Van Cleve ${ }^{5, *}$, Florence Débarre ${ }^{6}$, Maxime Deforet ${ }^{7}$, William R. Harcombe ${ }^{8}$, Jorge Peña ${ }^{9}$, Sam P. Brown ${ }^{10}$ and Michael E. Hochberg,

1. Department of Ecology and Evolutionary Biology, Yale University, New Haven, CT, USA

2. Microbial Sciences Institute, Yale University, West Haven, CT, USA

3. Santa Fe Institute, Santa Fe, NM, USA

4. Department of Mathematics, Umeå University, Umeå, Sweden

5. Department of Biology, University of Kentucky, Lexington, KY, USA

6. CIRB, Collège de France, CNRS, UMR 7241, Paris, France

7. Laboratoire Jean Perrin, CNRS, Sorbonne Université, Paris, France

8. Department of Ecology, Evolution and Behavior, University of Minnesota, Minneapolis, MN, USA

9. Institute for Advanced Study in Toulouse, Toulouse, France

10. University of Toulouse Capitole, Toulouse, France

11. School of Biological Sciences, Georgia Institute of Technology, Atlanta, GA, USA

12. Institut des Sciences de l'Evolution, CNRS UMR5554, University of Montpellier, Montpellier, France

${ }^{*}$ These authors contributed equally.

\# Correspondence: sylvie.estrela@yale.edu (S. Estrela); mhochber@univ-montp2.fr (M.E. Hochberg)

Keywords: social evolution; niche construction; microbes; dispersal; spatial structure; ecology

DOI:https://doi.org/10.1016/j.tree.2018.10.004

\section{Abstract}

By consuming and producing environmental resources, organisms inevitably change their habitat. The consequences of such environmental modifications can be detrimental or beneficial not only to the focal organism but also to other organisms sharing the same environment. Social evolution theory has been very influential in studying how social interactions mediated by public goods or bads evolve by emphasising the role of spatial structure. The environmental dimensions driving these interactions, however, are typically abstracted away. Here we propose a new, environmentally-mediated taxonomy of social behaviours where organisms are categorised by their production or consumption of environmental factors that help or harm others in the environment. We discuss microbial examples of our classification and highlight the importance of environmental intermediates more generally. 


\section{Why the environment matters for social evolution}

Organisms and their environment are inevitably coupled [1]. By consuming, transforming and producing environmental resources, individuals modify their habitat. The consequences of this change can be damaging to a focal individual and its neighbouring conspecifics, for example by producing harmful waste [2]. Conversely, environmental modification can be beneficial, for example when organisms invest in shelter or resource acquisition $[3,4]$.

Social evolution (see Glossary) theory is an important framework for understanding behaviours that impact the fitness of other individuals, potentially resulting in (social) trait evolution. Social evolution theory has been very influential in the study of interactions among conspecifics, but it has traditionally overlooked the role of environmental dimensions in mediating such interactions and their impacts on the environment. The classical taxonomy of social evolution, first introduced by Hamilton [5-7], considers fitness-affecting interactions between focal and recipient individuals. Helping interactions increase recipient fitness and can be either altruistic or mutualistic from the perspective of the focal actor, while harming interactions decrease recipient fitness and can be either selfish or spiteful when they increase or decrease, respectively, the fitness of the actor [5]. The mechanisms of helping and harming often involve an environmental currency - for example a physical refuge or a chemical attack - but these environmental dimensions are typically abstracted away so that the amount of 'help' ('harm') is simply defined by a proxy such as the number of 'helpers' ('harmers') in the neighbourhood of recipients $[8,9]$.

\section{Glossary}

Altruistic: trait that increases the fitness of others (recipients) but decreases the fitness of the focal organism (actor).

Cheats: organisms that benefit from other organism's costly behaviours, without contributing or contributing less.

Cooperation: (or helping) is social trait that increases the fitness of another individual (recipient).

Consumer-resource models: models that explicitly account for the dynamics of resources and of the organisms consuming those resources.

Dispersal: process by which one individual or a group of individuals move from one location to a new location. Dispersal can be active, through self-motility, or passive, due to external forces such as transportation through wind or water.

Mutualistic: trait that increases the fitness of both recipient and actor.

Selfish: trait that increases the fitness of the actor but decreases the fitness of the recipient

Spiteful: trait that decreases the fitness of both recipient and actor.

Lotka-Volterra models of competition: system of ordinary differential equations describing the dynamics of interacting populations (e.g. species). Common resources are not explicitly modeled and the effect that one species has on itself and on others is implicitly modeled with interaction coefficients.

Niche construction: concept that broadly describes any organismal trait (behaviour) that modifies its own and others environment. Such traits can have positive (constructive) or negative (destructive) effects on the environment.

Social evolution: area of evolutionary biology interested in explaining the evolution of traits by a focal individual (actor) that have an effect (positive or negative) on the fitness of other individuals (recipients), and that evolved, in part, because of these effects. 
The role of environmental intermediates in social interactions has received increasing attention in recent years due to the growing realization that explicitly considering environmental dimensions is critical to capturing realistic system complexity [1,8-12]. For example, if cooperation (or helping) among organisms improves the environment or prevents its degradation, then oscillations can emerge where cooperators and defectors alternately dominate as the environment cycles between replete and deplete states [11]. Thus, a more realistic and complex theory of social evolution that includes these important feedbacks needs to account for niche construction effects [1].

At present, theoretical work on environmentally-mediated social interactions has largely focused on simple non-spatial settings (except, notably, work on biofilms; e.g, [13-17]), while the broader field of social evolution has long recognized the importance of spatial structure and its role in generating genetic relatedness that can lead to the evolutionary success of cooperation and altruism (e.g., [5,18-20]). Mathematical models have shown the influence of spatial structure in determining the success of cooperative behaviours particularly in the face of non-linear group benefits (e.g., [21]), repeated interactions (e.g., [22,23]), or when individuals can move [24-28]. Yet, these models often do not explicitly consider environmental dimensions. The few models that do consider explicit environmental variables suggest that when cooperation improves the local environment, selection for cooperation can be very strong [29-32]. For example, Pepper and Smuts used an agent-based spatial model with conditional movement and environmental feedbacks to demonstrate that cooperation can be maintained in the presence of defection when cooperators improve the environment. Individuals are then less likely to leave locations with good environmental conditions [29].

Here, we seek to address questions concerning how different types of social behaviour can be maintained under the more realistic conditions of non-random (structured) interactions, explicit environmental currencies, and dispersal (or migration) of individuals (Figure 1). This is particularly relevant to cooperative behaviours, which have been shown to require one or more non-homogeneous selective structures to persist (e.g., [23,31,33-36]). For instance, can 'helper' organisms sufficiently co-localize with the products of their helping activities to survive free-loaders? Can 'harming' organisms escape the environmental damage they create? Given the complexity of spatial systems of interacting organisms and environments, the answers to these and other related questions are far from straightforward and require explicit mathematical models and controlled experiments (see Outstanding Questions).

\section{Environmental resources and the tragedy of the commons}

Social dilemmas are loosely characterized by the conflict between the individual costs (or benefits) of certain actions and the benefits (or costs) of those actions when performed by enough members of a collective. In many cases, these actions involve the production or consumption of environmental resources valuable for the survival and reproduction of individuals in a group, where groups are defined by the ability of individual members to access those resources. These accessible resources are typically depletable, such as glucose 
concentration in a bacterial batch culture, and are referred to as 'public goods' or 'common pool resources'. The social dilemma involved in maintaining public goods is often called the 'tragedy of the commons' [37]. The idea is that if members of a social group use a shared limiting resource ('the commons') selfishly without restraint, then the commons will be depleted and eventually destroyed. Since organisms across the tree of life exhibit a variety of mechanisms involved in the production, acquisition, or maintenance of environmental resources and public goods, they all potentially face this dilemma [38,39]. Yet, the degree to which evolution averts the tragedy of the commons is often a complex function of individual and collective interactions with the environment. Next, we present a classification of these interactions.

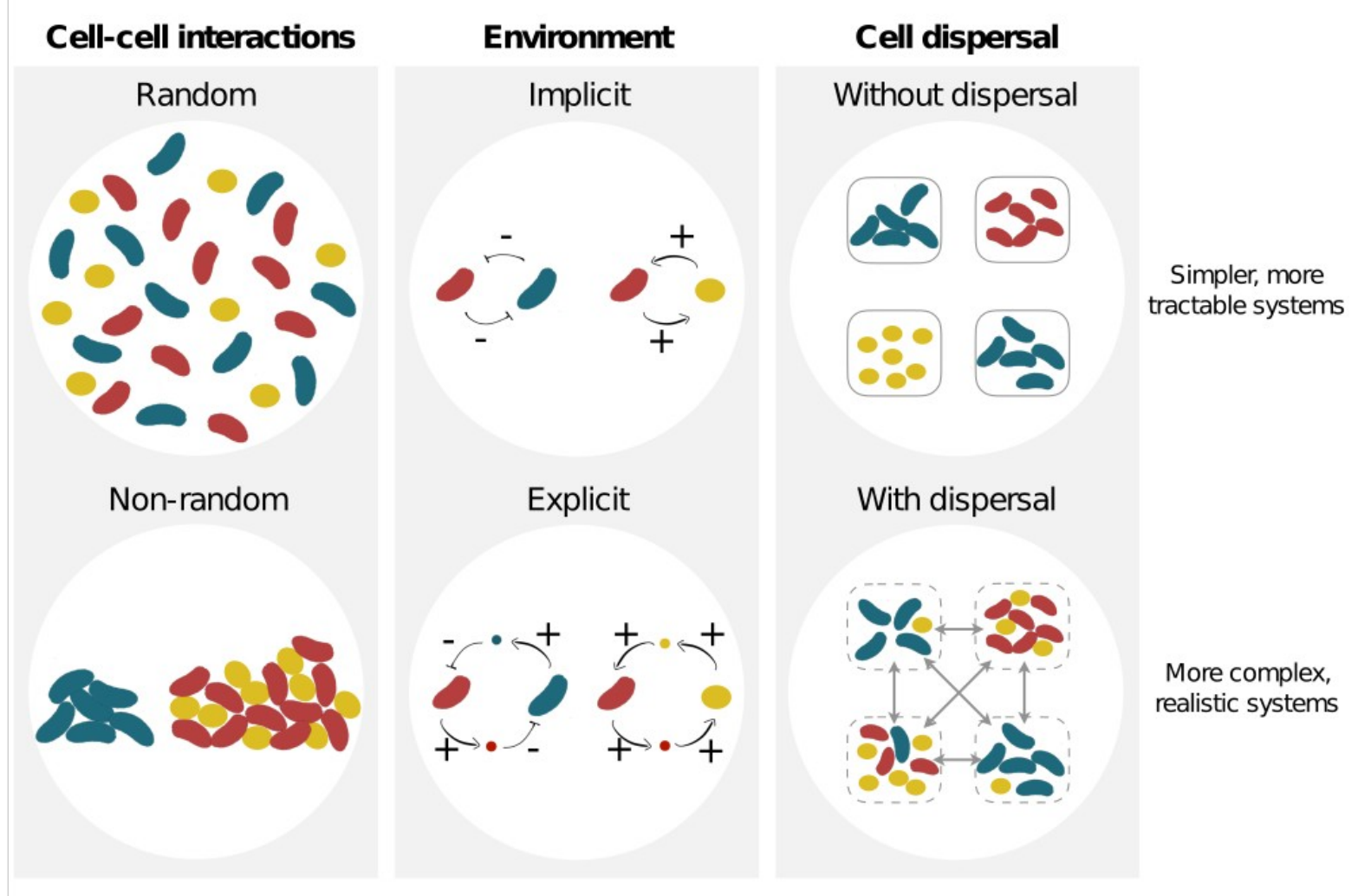

Figure 1. Variety of potential interactions between organisms and their environment. For simplicity, models of social evolution have often assumed that interactions between individuals are random, the physico-chemical environment is implicit, and/or individuals cannot move within a patch or disperse between patches (top scenarios). Here we focus on the more realistic conditions where interactions are non-random, the environmental dimension is explicit, and individuals can migrate (bottom scenarios) to investigate how these combined effects may influence the ecology and evolution of social behaviours. Note that we use the terms migration and dispersal interchangeably. 


\section{A classification of environmentally mediated social behaviours}

Here, we present a framework for classifying social dilemmas based on their environmental interactions that is inspired by Hamilton's individual-based classification [5-7]. Our first step is to recognize that helping and harming behaviours can be classified as one of two distinct environmental interactions: (1) when the actor produces a helpful or harmful environmental factor, and (2) when the actor consumes a harmful or helpful factor (Figure 2).

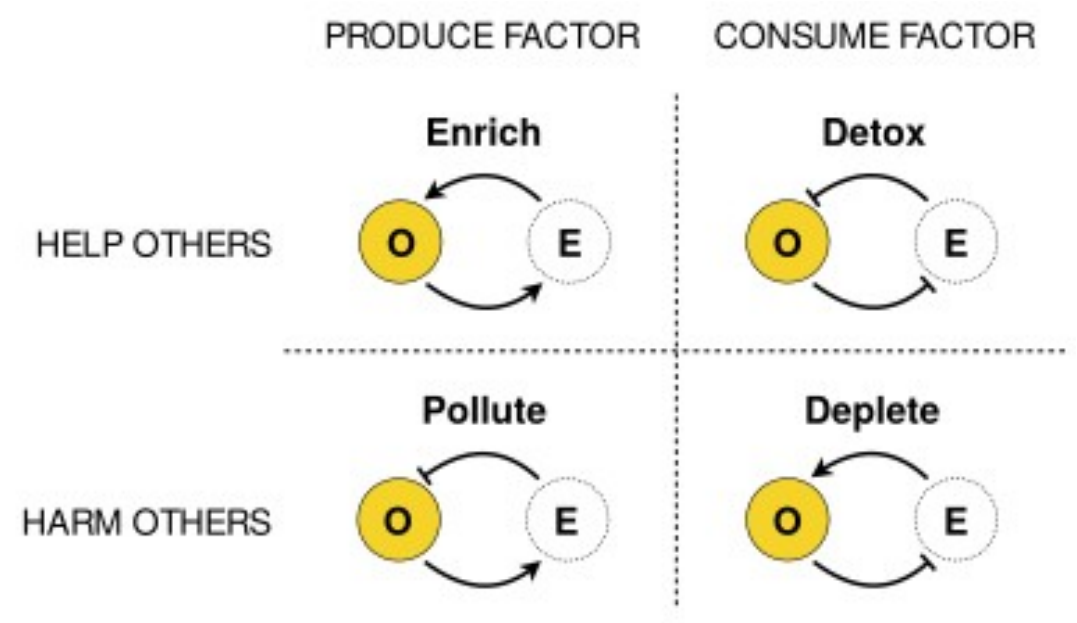

Figure 2. A classification of environmentally mediated social behaviours. Individual behaviours are categorised into helping / harming others, and also into producing / consuming an environmental factor, yielding a 4-way classification of behaviour. Schematic summaries illustrate the signs of reciprocal organism (O) - environment (E) interactions under each scenario (pointed arrow indicates a positive effect; flat arrow indicates a negative effect). Effects on organisms are generally measured as changes in direct fitness (e.g. growth rate, survival) and effects on environment are measured as changes in the density/amount of environmental factor. For each scenario, all individuals in the population are assumed to have the same behaviour, i.e. they act and are affected by the environment in the same way. Thus, helping (or harming) others means helping (or harming) other individuals of the same type.

In our classification, a focal individual (actor) that produces a collectively beneficial good is referred to as an 'enricher', whereas an actor that degrades a damaging environmental factor is a 'detoxifier'. Under the classical classification of social traits, both would be viewed as expressing a 'helping' behaviour (with further categorization as either altruistic or mutualistic depending on the degree to which the benefits return to the focal actor). Similarly, our new classification breaks down conventional 'harming' behaviours into 'pollute' and 'deplete', depending on whether the harming activity produces an environmental 'bad' or consumes a shared environmental 'good'. Thus, what appears as the same social behaviour under the classical taxonomy of social evolution, may actually be the result of opposing consume/ produce strategies when the environment is explicitly considered. Therefore, to understand how social traits mediated through the environment evolve, it is crucial to explicitly consider the contrast between producing and consuming environmental goods and bads, and how this 
influences the outcome of social dilemmas on both temporal and spatial scales. Below, we discuss examples of our 4-way classification with a focus on microbes.

\section{Microbial examples of the proposed classification}

Microbes affect each other in many ways. These effects may arise through direct physical contact (e.g. attachment, toxin injection), or indirectly by simply sharing the same chemical environment. Such environmental intermediates not only shape microbial composition ('who is there') but also the nature of their interaction ('what they do to each other'). Here, we go beyond the traditional 'helping' versus 'harming' classification of social behaviour. We discuss empirical and theoretical examples of our 4-way classification - enrich, detox, pollute, and deplete - to illustrate how considering explicit organism density and environmental dimensions can be important to understand the ecology and evolution of social dilemmas. Although the mechanisms of enrich, detox, pollute, and deplete are discussed separately, they are clearly often intertwined and interdependent, contributing to the rich and complex diversity of microbe-microbe metabolic interactions found in nature.

\section{Enrich}

Microbes can modify their environment by producing extracellular molecules that promote the growth of the focal individual and its neighbouring conspecifics. Examples include exoenzymes that break down complex substrates into simple digestible compounds (e.g. glycoside hydrolases and proteases) [40], scavenging molecules that bind specific compounds in the environment and transport them back into the cell (e.g. iron-scavenging siderophores $[41,42]$ ), and structural compounds in biofilms that protect microbes from chemical or biological attacks [43-45] or place them near other important resources such as oxygen [46]. In some cases, such exoenzymes benefit the focal producer and conspecifics by directly harming others such as antibiotics and toxins that kill or inhibit the growth of other interspecific organisms [47]. Production of such beneficial compounds are often costly and will only evolve if it sufficiently benefits the focal producer ('enricher'), as otherwise it would be selected against and eventually lost from the population. Any benefits leaked to other neighbouring organisms depend on how public or private these traits are.

\section{Detox}

A long-overlooked but increasingly important factor shaping microbial interactions is detoxification [48-58]. Examples include the production of enzymes such as beta-lactamases that degrade beta-lactam antibiotics [53,56], catalases that degrade hydrogen peroxide [51,58], and metal-chelating siderophores that reduce toxic metal levels [54,55]. Detoxification not only benefits the focal producer ('detoxifier') but also protects neighbouring cells living in the environment. Because enzyme production is generally costly, detoxification is expected to have evolved to benefit the producer, with the benefits to others depending on the privatization level of detoxification [49,59]. Detoxification can nevertheless 
also be 'incidental' (i.e., not selected for), such as when an organism feeds on compounds that are toxic to others but not to itself $[48,60]$.

\section{Pollute}

Microbial cells commonly excrete metabolic waste products such as lactic acid, acetate and hydrogen. These metabolic by-products may become toxic, especially when at high concentrations, suppressing the growth of not only the producer cell but also of any nearby susceptible cells (e.g., by changing the $\mathrm{pH}$ [61]). In some cases, however, such metabolic waste can be a resource for another species, allowing both polluters (waste producers) and detoxifiers (cross-feeders) to potentially benefit from the interaction if the mutual benefits of food for detoxification outweigh the costs of competition for shared resources [48]. Such syntrophic interactions are common between hydrogen-producing bacteria and methanogens. While the build-up of hydrogen inhibits the growth of the producing bacteria, methanogens are able to use hydrogen for growth, and thus keep hydrogen levels low, allowing their hydrogen-producing partner to grow [62,63].

\section{Deplete}

Competition over resources - occurring when organisms feed on shared, limiting resources (also known as 'exploitation competition') -is a key factor shaping microbial interactions [64]. Although resource competition is often initially weak, competition increases as cells grow and resources become depleted. In an unstructured environment with a single resource, theory predicts that the more competitive strain displaces the other strains, thus resulting in competitive exclusion [65]. However, the presence of trade-offs [66,67], such as 'fastgrowing but low-yield' vs 'slow-growing but high-yield', can promote species coexistence even when organisms compete for a single resource. From a social evolution perspective, a slow-growing and high-yield strategy can be viewed as a form of cooperation because resources are used in a more prudent way compared to a fast-growing and low-yield strategy. Thus, the evolutionary dilemma is how to maintain prudent (cooperative) resource use in the face of rapacious (non-cooperative) individuals that eventually deplete the commons. A solution to this dilemma is spatial structure that enables cooperators to preferentially interact with other cooperators and remain protected from the local effects of non-cooperators [13,6870].

\section{The value of explicit environmental variables}

Models of resource competition have often assumed that resources are implicit (e.g., LotkaVolterra models of competition, [71]) or explicit but with a single resource pool (e.g. classic consumer-resource models with a single limiting resource, [72]). Microbial cells, however, are often surrounded by a variety of genotypes with different resource-use strategies and diverse resource pools (supplied exogenously and/or as by-products of cell metabolism). Classic population ecology models or resource-based models with a single resource can therefore lead to an oversimplified depiction of organism-organism interactions and, in some 
cases, fail to predict the dynamics of microbial interactions [73,74]. Work combining these and other explicit metabolic models with spatially-explicit environmental models [75-77] have recently received more attention and promise to yield a wealth of insights into more realistic representations of ecological interactions in nature.

\section{Environment-mediated organism-organism interactions}

If we abstract away the environment and consider two organisms and the various positive and negative interactions they can exert on themselves and each other, we find ten possible scenarios after removing symmetries (Figure 3). Six of these interaction scenarios can occur via a single environmental compound (A, D-G,J). Interestingly, some reduced (environmentally-implicit) systems map to two different environmentally-mediated social interactions A,D,E,J), while others map to only one (F,G). The other four scenarios, however, are impossible to achieve with only one environmental intermediate (B,C,H,I). To see this, consider scenario $\mathrm{B}$ in which one organism $\left(\mathrm{O}_{1}\right)$ positively affects itself and the other organism $\left(\mathrm{O}_{2}\right)$. This could occur through the removal of a toxic product (detox) or the production of a beneficial product (enrich). In addition, organism $\mathrm{O}_{2}$ interacts positively with $\mathrm{O}_{1}$ but negatively with itself (e.g. by producing a waste product that is toxic to itself but on which $\mathrm{O}_{1}$ can feed, or by feeding on a compound that is toxic to $\mathrm{O}_{1}$ ). A simple ' $\mathrm{O}_{1}-\mathrm{E}_{1}-\mathrm{O}_{2}$ ' schematic is limited because it cannot capture the possibility that the single environmental compound $\left(E_{1}\right)$ that helped $\mathrm{O}_{1}$ and helped $\mathrm{O}_{2}$ can also help $\mathrm{O}_{1}$ and hurt $\mathrm{O}_{2}$. Therefore we need a second environmental factor $\left(\mathrm{E}_{2}\right)$ to be able to capture such scenarios.

Furthermore, the mappings from the reduced system to the environmentally-explicit system are not always identical. For instance, scenario $\mathrm{J}$ in the reduced system corresponds to a case where each organism negatively affects itself and the other organism. In the environmentallyexplicit model, this can be either deplete/deplete or pollute/pollute. In the case of deplete/deplete, both organisms consume the same resource and hence negatively affect themselves and each other by removing the resource. In the case of pollute/pollute, both organisms produce the same waste product that negatively affects everyone. Importantly, these two scenarios produce different dynamical models, especially regarding the dynamics of E. It is also worth noting that while some implicit models where $\mathrm{O}_{1}$ and $\mathrm{O}_{2}$ have the same strategy lead to explicit models where $\mathrm{O}_{1}$ and $\mathrm{O}_{2}$ also have the same strategy (J), in other cases $\mathrm{O}_{1}$ and $\mathrm{O}_{2}$ must have different strategies (F, G). 

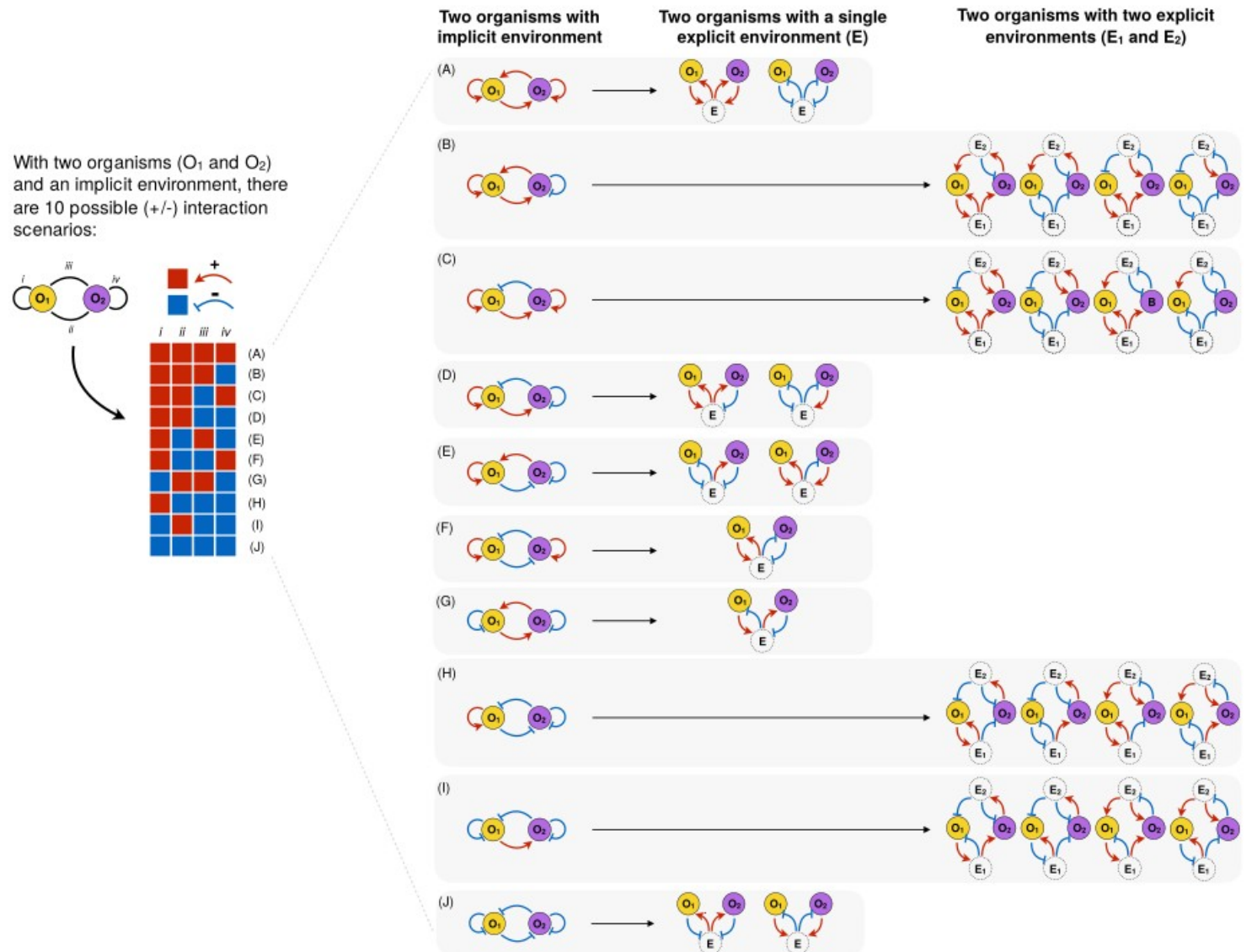

Figure 3. Environment-mediated organism-organism interactions. Illustrated are the possible combinations of positive (red) and negative (blue) interaction scenarios between two organisms $\left(\mathrm{O}_{1}\right.$ and $\left.\mathrm{O}_{2}\right)$ from different types when the environment is implicit and when taking into account the effect of each organism on themselves and individuals of the same type (illustrated as an arrow end pointing towards self) and the effect on others (pointing towards the other type). Note that here we remove symmetries, that is, when species $\mathrm{O}_{1}$ and $\mathrm{O}_{2}$ are interchangeable. Six of the reduced systems can be used to describe all ten unique scenarios involving a single environmental intermediate $\left(E_{1}\right)$ and combinations of the environmental modifications enrich, detox, pollute, deplete (A, D-G, J). The other four reduced systems cannot be described with a single environmental factor and at least two are needed (B, C, H, I). Note that here we only represent environmental variables that directly mediate the interaction between the two organisms. Environmental factors that affect the growth of one organism but not the other are omitted.

\section{Spatial and temporal dynamics of environmentally-mediated social dilemmas}

While it is well known in social evolution theory that the spatial scale at which cooperation and conflict over resources occur can have a large impact on evolved levels of cooperation [78-81], it is possible that unexpected social evolutionary effects emerge as a consequence of interactions between environmental, spatial, and temporal parameters. To illustrate these 
possibilities, we describe below how the viscosity (spatial scale) and persistence (temporal scale) of environmental factors can affect the evolution of cooperative traits, and in turn, the spatial arrangement of cooperators and non-cooperators.

\section{Environmental viscosity: cell dispersal and exoproduct diffusion}

Environmental mediation allows for social interactions to take place at larger spatial scales via diffusible compounds. Whether or not spatial dynamics favour enriching strategies, depends on environmental viscosity and its effect on the levels of cell dispersal and public goods diffusion [80,82]. In the case of high viscosity where both cell dispersal and public goods diffusion are limited, the benefits of public goods can be channeled between adjacent, and likely related organisms. This acts to privatize the produced goods and can promote cooperation (see e.g., [82,83]). However, if viscosity is such that cell dispersal is limited while public goods diffusion remains high, then the spatial scale over which cooperative interactions occur becomes larger than the spatial scale of competition [84] and viscosity will not favour the evolution of cooperation (see e.g., [85]).

The interplay of social interactions and environmental viscosity has received particular attention in the study of microbial biofilms [17]. To capture realistic features of microbial biofilms as well as the chemical-physical properties of the environment spatially and mechanistically explicit individual-based models have shown to be a powerful approach [15]. Such models can provide important insights into how factors such as exoproduct diffusion, population growth, and social interactions affect the eco-evolutionary dynamics of these spatially-structured systems $[17,86]$. For instance, the extracellular matrix that generates biofilms was initially thought of as a public good that could be exploited by non-producers. However, individual-based models that incorporated nutrient diffusion and cell limited dispersal demonstrated that polymer secretion is often a competitive strategy that lifts producer genotypes into favourable environments while suffocating non-producer genotypes [14]. Further, individual-based models have revealed how social interactions shape the arrangement of genotypes, and therefore, selection in structured systems. While cells with cooperative genotypes will mix as they grow and divide, competing genotypes become segregated as a result of growth $[87,88]$. This can lead to social insulation of cooperative genotypes from cheating mutants and enhance selection for cooperation [16,89].

\section{Environmental persistence: organism lifespan and exoproduct durability}

Another important consequence of environmental mediation is that when modified environmental factors persist for long enough, their effects can be transmitted across generations $[8,10]$ (so that there is ecological inheritance sensu $[1,90]$ ). This means that actors interact not only with existing relatives, but also with relatives living in the future who are not in direct competition with actors in the present [91,92]. This decouples kin selected benefits and costs due to kin competition and can increase selection pressure on helping traits, including altruistic behaviours that would be selected against when actors and recipients only interact during the same generation [91]. For durable public goods that are non-rival (i.e. not consumed by use), investments by earlier generations can lead to loss of cooperative 
genotypes in subsequent generations - unless cooperative behaviours are under regulatory control $[8,10]$. For microbial interactions, these observations suggest that not only the viscosity of the medium is important, but also the durability of secreted compounds relative to the lifespan of organisms. In the case where exoproduct durability is sufficiently larger than the lifespan of the focal producer, ecological inheritance can operate, thus paving the way for posthumous niche construction [92].

\section{Environmentally-mediated conditional dispersal}

The production and consumption of environmental factors (as in the enrich, detox, pollute, deplete scenarios) are one of two ways organisms can engage in niche-constructing activities. The other way is the active movement of organisms within a patch and dispersal between patches. By dispersing, an individual necessarily changes the patch from which it dispersed, and the patch to which it migrates [1]. One inclusive fitness benefit of dispersal is the ability of an individual to avoid competing for resources with its relatives in its natal patch. Though this topic has received considerable theoretical attention [27,93-100], previous work mostly considers the environment implicitly and does not detail the helpful or harmful environmental factors that affect survival and reproduction (i.e., our classification above). One crucial consequence of omitting these environmental factors is the inability to consider how dispersal can evolve to be conditional on the state of the local environment [101], which is itself affected by organismal niche construction. Since many social interactions can be produced by multiple explicit interactions with the environment (see Figure 3), the effect of dispersal conditional on environmental state is likely to depend on these explicit interactions.

To see how dispersal conditional on environmental variables may be important, consider scenario $\mathrm{J}$ in Figure 3, whose implicit representation consists of negative effects of each organism on itself and on the other organism. Scenario J can be represented by two explicit environmental interactions: (i) both organisms negatively affect the environmental compound which in turn positively affects them, as occurs in a 'deplete' scenario, or (ii) both organisms positively affect the environment which negatively affects them, as occurs in a 'pollute' scenario. Organismal growth rates increase with greater concentrations of the environmental compound in the 'deplete' scenario and decrease in the 'pollute' scenario. Thus, all else equal, dispersal rates away from the current patch may evolve to be higher for low concentrations of the environmental product in the 'deplete' scenario and lower for high concentrations of the product in the 'pollute' scenario (Box 1). Thus, a single implicit social interaction can generate different dispersal mechanisms depending on the explicit environmental interactions. For a more complex scenario, see Box 1.

Further, dispersal conditional on explicit environmental variables may affect the conditions under which different kinds of social behaviours evolve. For example, models have shown that cooperative interactions that increase group survival or carrying capacity can easily evolve [102,103]. In effect, these cases represent a type of 'enrich' scenario whereby cooperators create larger and more persistent groups. However, if dispersal is allowed to be 
conditional on local resource concentrations, then non-cooperators are no longer so easily trapped in unproductive groups that go extinct and might preferentially disperse from lowresource patches to high-resource patches with more cooperators. Such conditional dispersal would likely erode the chance that cooperators enrich the environment for other cooperators and diminish selection for cooperation in these scenarios. Interestingly, there also might be selection for conditional dispersal of cooperators away from low-resource patches to highresource patches. Since high-resource concentrations typically cannot be generated by groups composed mostly of non-cooperators, they might serve as an environmental cue of the presence of other cooperators and could create the kind of positive assortment among cooperators necessary to maintain them in the presence of non-cooperators [23,31]. In fact, Pepper and Smuts [29] studied this kind of conditional dispersal and found that it can generate significant assortment between cooperators. More generally, conditional dispersal might either enhance or erode cooperation depending on the details of the environmental interactions. Thus, not including explicit environmental interactions could exclude important ecological and evolutionary dynamics in social dilemmas.

\section{Box 1. The value of explicit environment representation in the evolution of dispersal}

The interplay between dispersal and environmentally-mediated social dilemmas can benefit from explicit representations of the environment in theoretical models. For example, consider scenario $\mathrm{F}$ in Figure 3. In an implicit representation of the environment, organisms $\mathrm{O}_{1}$ and $\mathrm{O}_{2}$ appear identical in kind: both help themselves and harm the other. Now suppose that each organism can disperse to a new environment. Under the implicit representation, there is no suggestion that $\mathrm{O}_{1}$ and $\mathrm{O}_{2}$ would follow different dispersal strategies. Yet, an explicit representation of the environment shows that they are affected by the environment differently: one enriches the environment while the other detoxifies it. Thus, we would expect each organism to follow a different dispersal strategy as a function of environmental state: the enrich organism has a higher growth rate in higher concentrations of $E$ and so should remain in high E patches (lower dispersal rate from such patches) while the detox organism is harmed by higher concentrations of $\mathrm{E}$ and so should move away from high $\mathrm{E}$ patches (high dispersal rate) (Figure IA). In a sense, this agrees with the implicit environmental model in that the two organisms should disperse away from one another since they harm each other. Yet, the explicit environmental model suggests a mechanism by which a patchy environment of high/low concentrations of $\mathrm{E}$ might facilitate a corresponding spatial assortment of the organisms. Now let's turn to scenario J in Figure 3. Again, in an implicit representation of the environment, organisms $\mathrm{O}_{1}$ and $\mathrm{O}_{2}$ appear identical in kind: both harm themselves and harm the other. This time, however, the two organisms interact with the environment in the same way and two different environmentally-mediated interactions are possible: either deplete/deplete or pollute/pollute. We therefore expect each organism to adopt the same dispersal strategy. But importantly, which strategy they adopt - higher or lower dispersal rate- depends on how the explicit environment is represented (Figure IB). Such a contrast 
would be difficult to capture with an environmentally implicit model. This is also the case in scenario $\mathrm{E}$ in Figure 3, in which the two organisms interact with the environment in a different way, and therefore should adopt opposite dispersal strategies. But whether each organism should stay or leave a patch with a particular concentration of $\mathrm{E}$ is determined by how the environment is represented (Figure IC). Together, these examples highlight how considering both environmental and social conditions explicitly can provide important insights for understanding and predicting the evolution of conditional dispersal.

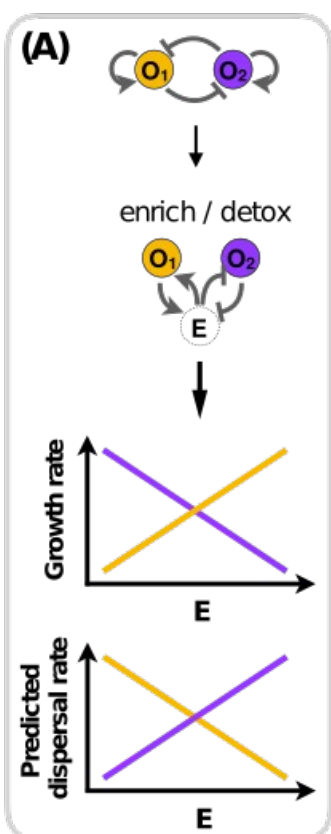

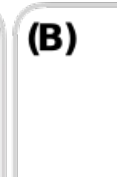

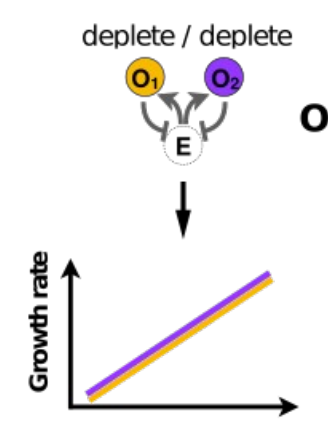

$\mathbf{E}$

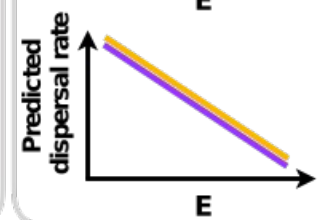

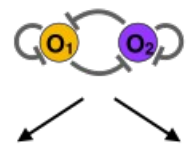

pollute / pollute
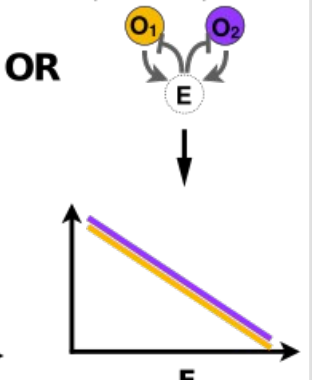

$\mathbf{E}$

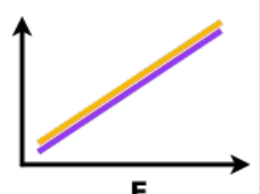

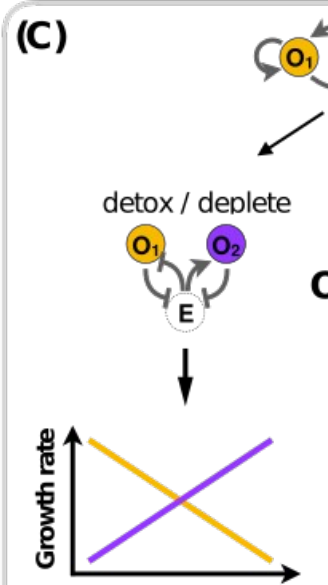

E

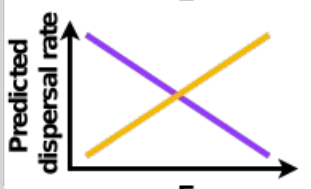

$\mathbf{E}$
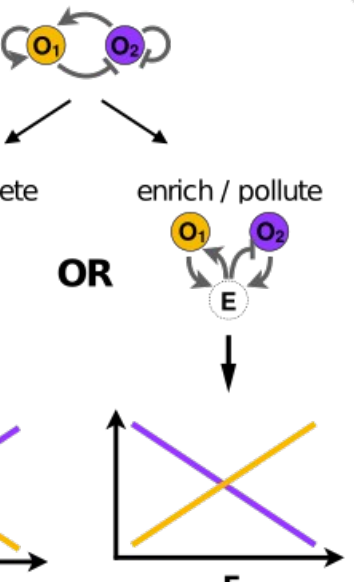

E

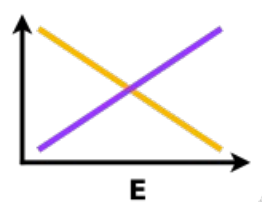

Figure I. The value of explicit environment representation for predicting the evolution of dispersal. Plots show the predicted growth rates and dispersal probabilities of two organisms $\left(\mathrm{O}_{1}\right.$ and $\left.\mathrm{O}_{2}\right)$ interacting through a shared environmental factor $(\mathrm{E})$ for three of the scenarios represented in Figure 3. The lines are simply meant to represent the direction of the trend (increasing/ decreasing) not the form (depicted as linear, for simplicity). Here dispersal probability of $\mathrm{O}_{1}$ (or $\mathrm{O}_{2}$ ) means an organism's probability of moving away from a patch with density E.

\section{Concluding remarks}

The typology we describe in Figure 2 aims to catalyse a better mechanistic understanding of the ecological and evolutionary dynamics of environmentally mediated social dilemmas. Nevertheless, the simple typology of social dilemmas initiated by Hamilton that classifies behaviours strictly based on their fitness effects is still an important conceptual tool [5]. It produces many insights including the role of genetic identity and co-ancestry in the evolution of altruism [5] and the effect of local competition for resources and other demographic forces in altering selection for or against cooperation [18,19,102]. Therefore, a crucial task is to develop formal mathematical connections between the environmentally-mediated and classical social dilemma typologies. 
Under some circumstances, environmentally implicit approaches will accurately represent social dilemmas and their dynamics. Evidently, this is increasingly the case as habitats become unstructured, social interactions are more direct and less mediated by elements of the environment (as occurs with mechanisms requiring direct cell-to-cell contact, e.g., competitor killing via the type VI secretion systems (T6SS) [104]), and when the environmental dimension is coupled to producers (e.g., when there is a fast turnover of the environmental currency and/or a short lag between changes in focal producer and public good/bad densities [8]). However, we suspect that such simplicity, even if common, is not representative of many environments. Thus, a more explicit framework such as the one presented here is necessary for structured environments and environmentally-mediated social interactions. Attaining both generality and accuracy in such a framework will be challenging, both because of the stunning diversity of social systems [105] and their complex associations with the environment [1]. Nevertheless, the ubiquitous importance of social interactions and how dilemmas are mediated by ecology and evolution argue for an environmentally-inclusive framework, and future investigation using mathematical, behavioural, molecular and genomic tools. Although our examples focus on microbial systems, we believe that our four-way classification will also be useful for understanding interactions mediated through the environment in other systems, like social animals, and humans.

\section{Acknowledgements}

We wish to thank the Santa Fe Institute for hosting the two working groups in 2016 and 2017, during which this research was conducted, and four anonymous reviewers for helpful suggestions. FD received funding from ANR (ANR-14-ACHN-0003). JP gratefully acknowledges financial support from the ANR-Labex IAST. MEH thanks the McDonnell Foundation (Studying Complex Systems Research Award No. 220020294).

\section{References}

1 Odling-Smee, F.J. et al. (2003) Niche construction: the neglected process in evolution, Princeton University Press.

2 Bédhomme, S. et al. (2005) Pollution by conspecifics as a component of intraspecific competition among Aedes aegypti larvae. Ecol. Entomol. 30, 1-7

3 Crespi, B.J. (2001) The evolution of social behavior in microorganisms. Trends Ecol. Evol. 16, 178-183

4 West, S.A. et al. (2006) Social evolution theory for microorganisms. Nat. Rev. Microbiol. 4, 597-607

5 Hamilton, W.D. (1964) The genetical evolution of social behaviour. I. J. Theor. Biol. $7,1-16$ 
6 Hamilton, W.D. (1964) The genetical evolution of social behaviour. II. J. Theor. Biol. $7,17-52$

7 Hamilton, W.D. (1970) Selfish and spiteful behaviour in an evolutionary model. Nature 228, 1218-1220

8 Brown, S.P. and Taddei, F. (2007) The Durability of Public Goods Changes the Dynamics and Nature of Social Dilemmas. PLoS ONE 2, e593

9 McNally, L. and Brown, S.P. (2015) Building the microbiome in health and disease: niche construction and social conflict in bacteria. Philos. Trans. R. Soc. Lond., B, Biol. Sci. 370, 20140298

10 Kümmerli, R. and Brown, S.P. (2010) Molecular and regulatory properties of a public good shape the evolution of cooperation. Proc. Natl. Acad. Sci. U.S.A. 107, 18921-18926

11 Weitz, J.S. et al. (2016) An oscillating tragedy of the commons in replicator dynamics with game-environment feedback. Proc. Natl. Acad. Sci. U.S.A. 113, E7518-E7525

12 Benincà, E. et al. (2015) Species fluctuations sustained by a cyclic succession at the edge of chaos. Proc. Natl. Acad. Sci. U.S.A. 112, 6389-6394

13 Kreft, J.U. (2004) Biofilms promote altruism. Microbiology 150, 2751-2760

14 Xavier, J.B. and Foster, K.R. (2007) Cooperation and conflict in microbial biofilms. Proc. Natl. Acad. Sci. U.S.A. 104, 876-881

15 Nadell, C.D. et al. (2010) Emergence of spatial structure in cell groups and the evolution of cooperation. PLoS Comput. Biol. 6, e1000716

16 Mitri, S. et al. (2011) Social evolution in multispecies biofilms. Proc. Natl. Acad. Sci. U.S.A. 108, 10839-10846

17 Nadell, C.D. et al. (2016) Spatial structure, cooperation and competition in biofilms. Nat. Rev. Microbiol. 14, 589-600

18 Lehmann, L. and Rousset, F. (2010) How life history and demography promote or inhibit the evolution of helping behaviours. Philos. Trans. R. Soc. Lond., B, Biol. Sci. 365, 2599-2617

19 Taylor, P.D. (1992) Altruism in viscous populations - an inclusive fitness model. Evol. Ecol. 6, 352-356

20 Lehmann, L. et al. (2007) The evolution of helping and harming on graphs: the return of the inclusive fitness effect. J. Evol. Biol. 20, 2284-2295

21 Peña, J. et al. (2015) Evolutionary dynamics of collective action in spatially structured populations. J. Theor. Biol. 382, 122-136

22 Akçay, E. and van Cleve, J. (2012) Behavioral responses in structured populations pave the way to group optimality. Am. Nat. 179, 257-269 
23 van Cleve, J. and Akçay, E. (2014) Pathways to social evolution: reciprocity, relatedness, and synergy. Evolution 68, 2245-2258

24 Hauert, C. and Doebeli, M. (2004) Spatial structure often inhibits the evolution of cooperation in the snowdrift game. Nature 428, 643-646

25 Aktipis, C.A. (2004) Know when to walk away: contingent movement and the evolution of cooperation. J. Theor. Biol. 231, 249-260

26 Aktipis, C.A. (2011) Is cooperation viable in mobile organisms? Simple Walk Away rule favors the evolution of cooperation in groups. Evol. Hum. Behav. 32, 263-276

27 Hochberg, M.E. et al. (2008) The coevolution of cooperation and dispersal in social groups and its implications for the emergence of multicellularity. BMC Evol. Biol. 8, 238

28 Parvinen, K. (2013) Joint evolution of altruistic cooperation and dispersal in a metapopulation of small local populations. Theor. Popul. Biol. 85, 12-19

29 Pepper, J.W. and Smuts, B.B. (2002) A mechanism for the evolution of altruism among nonkin: Positive assortment through environmental feedback. Am. Nat. 160, 205-213

30 Pepper, J.W. (2007) Simple models of assortment through environmental feedback. Artif. Life 13, 1-9

31 Lehmann, L. and Keller, L. (2006) The evolution of cooperation and altruism - A general framework and a classification of models. J. Evol. Biol. 19, 1365-1376

32 Kéfi, S. et al. (2008) Evolution of local facilitation in arid ecosystems. Am. Nat. 172, E1-17

33 West, S.A. et al. (2007) Evolutionary Explanations for Cooperation. Curr. Biol. 17, R661-R672

34 Nowak, M.A. (2006) Five Rules for the Evolution of Cooperation. Science 314, 15601563

35 Fletcher, J.A. and Doebeli, M. (2009) A simple and general explanation for the evolution of altruism. Proc. Biol. Sci. 276, 13-19

36 Frank, S.A. (1994) Genetics of mutualism: the evolution of altruism between species. J. Theor. Biol. 170, 393-400

37 Hardin, G. (1968) The tragedy of the commons. Science 162, 1243-1248

38 Rankin, D.J. et al. (2007) The tragedy of the commons in evolutionary biology. Trends Ecol. Evol. 22, 643-651

39 Levin, S.A. (2014) Public goods in relation to competition, cooperation, and spite. Proc. Natl. Acad. Sci. U.S.A. 111, 10838-10845

40 Rakoff-Nahoum, S. et al. (2016) The evolution of cooperation within the gut microbiota. Nature 533, 255-259 
41 Griffin, A.S. et al. (2004) Cooperation and competition in pathogenic bacteria. Nature 430, 1024-1027

42 Cordero, O.X. et al. (2012) Public good dynamics drive evolution of iron acquisition strategies in natural bacterioplankton populations. Proc. Natl. Acad. Sci. U.S.A. 109, 2005920064

43 Nadell, C.D. et al. (2015) Extracellular matrix structure governs invasion resistance in bacterial biofilms. ISME J. 9, 1700-1709

44 Vidakovic, L. et al. (2018) Dynamic biofilm architecture confers individual and collective mechanisms of viral protection. Nat. Microbiol. 3, 26-31

45 Kovacs, A.T. et al. (2012) The protective layer of biofilm: a repellent function for a new class of amphiphilic proteins. Mol. Microbiol. 85, 8-11

46 Rainey, P.B. and Rainey, K. (2003) Evolution of cooperation and conflict in experimental bacterial populations. Nature 425, 72-74

47 Riley, M.A. et al. (1999) The ecological role of bacteriocins in bacterial competition. Trends Microbiol. 7, 129-133

48 Estrela, S. et al. (2012) From metabolism to ecology: cross-feeding interactions shape the balance between polymicrobial conflict and mutualism. Am. Nat. 180, 566-576

49 Estrela, S. et al. (2016) Private benefits and metabolic conflicts shape the emergence of microbial interdependencies. Environ. Microbiol. 18, 1415-1427

50 Yurtsev, E.A. et al. (2016) Oscillatory dynamics in a bacterial cross-protection mutualism. Proc. Natl. Acad. Sci. U.S.A. 113, 6236-6241

51 Morris, J.J. et al. (2011) Dependence of the cyanobacterium Prochlorococcus on hydrogen peroxide scavenging microbes for growth at the ocean's surface. PLoS ONE 6, e16805

52 Sorg, R.A. et al. (2016) Collective Resistance in Microbial Communities by Intracellular Antibiotic Deactivation. PLoS Biol. 14, e2000631

53 Medaney, F. et al. (2016) Live to cheat another day: Bacterial dormancy facilitates the social exploitation of $\beta$-lactamases. ISME J. 10, 778-787

54 O’Brien, S. et al. (2018) No effect of intraspecific relatedness on public goods cooperation in a complex community. Evolution 72, 1165-1173

55 O’Brien, S. et al. (2014) Social evolution of toxic metal bioremediation in Pseudomonas aeruginosa. Proc. R. Soc. B Biol. Sci. 281, 20140858

56 Frost, I. et al. (2018) Cooperation, competition and antibiotic resistance in bacterial colonies. ISME J. 12, 1582-1593 
57 Estrela, S. and Brown, S.P. (2018) Community interactions and spatial structure shape selection on antibiotic resistant lineages. PLoS Comput. Biol. 14, e1006179

58 Morris, J.J. et al. (2014) Coexistence of evolving bacteria stabilized by a shared black queen function. Evolution 68, 2960-2971

59 Morris, J.J. (2015) Black Queen evolution: the role of leakiness in structuring microbial communities. Trends Genet. 31, 475-482

60 Cowan, S.E. et al. (2000) Commensal interactions in a dual-species biofilm exposed to mixed organic compounds. Appl. Environ. Microbiol. 66, 4481-4485

61 Ratzke, C. and Gore, J. (2018) Modifying and reacting to the environmental pH can drive bacterial interactions. PLoS Biol. 16, e2004248

62 Morris, B.E.L. et al. (2013) Microbial syntrophy: interaction for the common good. FEMS Microbiol. Rev. 37, 384-406

63 Hillesland, K.L. and Stahl, D.A. (2010) Rapid evolution of stability and productivity at the origin of a microbial mutualism. Proc. Natl. Acad. Sci. U.S.A. 107, 2124-2129

64 Ghoul, M. and Mitri, S. (2016) The Ecology and Evolution of Microbial Competition. Trends Microbiol. 24, 833-845

65 Hardin, G. (1960) The competitive exclusion principle. Science 131, 1292-1297

66 Bohannan, B.J.M. et al. (2002) Trade-offs and coexistence in microbial microcosms. Ant. Van Leeuw. 81, 107-115

67 Litchman, E. et al. (2015) Microbial resource utilization traits and trade-offs: implications for community structure, functioning, and biogeochemical impacts at present and in the future. Front. Microbiol. 6, 65

68 Pfeiffer, T. et al. (2001) Cooperation and competition in the evolution of ATPproducing pathways. Science 292, 504-507

69 Kerr, B. et al. (2006) Local migration promotes competitive restraint in a hostpathogen“tragedy of the commons.” Nature 442, 75-78

70 Nahum, J.R. et al. (2011) Evolution of restraint in a structured rock-paper-scissors community. Proc. Natl. Acad. Sci. U.S.A. 108, 10831-10838

71 Volterra, V. (1928) Variations and Fluctuations of the Number of Individuals in Animal Species living together. ICES J Mar. Sci. 3, 3-51

72 Chesson, P. (1990) MacArthur's consumer-resource model. Theor Popul Biol 37, 2638

73 Momeni, B. et al. (2017) Lotka-Volterra pairwise modeling fails to capture diverse pairwise microbial interactions. elife 6, 205 
74 Goldford, J.E. et al. (2018) Emergent simplicity in microbial community assembly. Science 361, 469-474

75 Harcombe, W.R. et al. (2014) Metabolic resource allocation in individual microbes determines ecosystem interactions and spatial dynamics. Cell Reports 7, 1104-1115

76 Chacón, J.M. et al. (2018) The spatial and metabolic basis of colony size variation. ISME J. 12, 669-680

77 Xavier, J.B. et al. (2005) A framework for multidimensional modelling of activity and structure of multispecies biofilms. Environ. Microbiol. 7, 1085-1103

78 Allison, S.D. (2005) Cheaters, diffusion and nutrients constrain decomposition by microbial enzymes in spatially structured environments. Ecol. Lett. 8, 626-635

79 Driscoll, W.W. and Pepper, J.W. (2010) Theory for the evolution of diffusible external goods. Evolution 64, 2682-2687

80 Allen, B. et al. (2013) Spatial dilemmas of diffusible public goods. elife 2, e01169

81 Dobay, A. et al. (2014) Interaction effects of cell diffusion, cell density and public goods properties on the evolution of cooperation in digital microbes. J. Evol. Biol. 27, 18691877

82 Kümmerli, R. et al. (2014) Habitat structure and the evolution of diffusible siderophores in bacteria. Ecol. Lett. 17, 1536-1544

83 Kümmerli, R. et al. (2009) Viscous medium promotes cooperation in the pathogenic bacterium Pseudomonas aeruginosa. Proc. Biol. Sci. 276, 3531-3538

84 Platt, T.G. and Bever, J.D. (2009) Kin competition and the evolution of cooperation. Trends Ecol. Evol. 24, 370-377

85 Le Gac, M. and Doebeli, M. (2010) Environmental viscosity does not affect the evolution of cooperation during experimental evolution of colicigenic bacteria. Evolution 64, $522-533$

86 Steenackers, H.P. et al. (2016) Experimental evolution in biofilm populations. FEMS Microbiol. Rev. 40, 373-397

87 Estrela, S. and Brown, S.P. (2013) Metabolic and demographic feedbacks shape the emergent spatial structure and function of microbial communities. PLoS Comput. Biol. 9, e1003398

88 Momeni, B. et al. (2013) Strong inter-population cooperation leads to partner intermixing in microbial communities. elife 2, e00230

89 Harcombe, W.R. et al. (2016) Adding biotic complexity alters the metabolic benefits of mutualism. Evolution 70, 1871-1881

90 Odling-Smee, F.J. et al. (1996) Niche Construction. Am. Nat. 147, 641-648 
91 Lehmann, L. (2007) The evolution of trans-generational altruism: Kin selection meets niche construction. J. Evol. Biol. 20, 181-189

92 Lehmann, L. (2008) The adaptive dynamics of niche constructing traits in spatially subdivided populations: evolving posthumous extended phenotypes. Evolution 62, 549-566

93 Hamilton, W.D. and May, R.M. (1977) Dispersal in stable habitats. Nature 269, 578581

94 Taylor, P.D. and Frank, S.A. (1996) How to Make a Kin Selection Model. J. Theor. Biol. 180, 27-37

95 Gandon, S. and Michalakis, Y. (1999) Evolutionarily Stable Dispersal Rate in a Metapopulation with Extinctions and Kin Competition. J. Theor. Biol. 199, 275-290

96 Perrin, N. and Lehmann, L. (2001) Is sociality driven by the costs of dispersal or the benefits of philopatry? A role for kin-discrimination mechanisms. Am. Nat. 158, 471-483

97 Le Galliard, J.F. et al. (2005) Adaptive evolution of social traits: Origin, trajectories, and correlations of altruism and mobility. Am. Nat. 165, 206-224

98 Mouden, El, C. and Gardner, A. (2008) Nice natives and mean migrants: the evolution of dispersal-dependent social behaviour in viscous populations. J. Evol. Biol. 21, 1480-1491

99 Lehmann, L. and Perrin, N. (2002) Altruism, dispersal, and phenotype-matching kin recognition. Am. Nat. 159, 451-468

100 Mullon, C. et al. (2018) Social polymorphism is favoured by the co-evolution of dispersal with social behaviour. Nat. Ecol. Evol. 2, 132-140

101 Rodrigues, A.M.M. and Johnstone, R.A. (2014) Evolution of positive and negative density-dependent dispersal. Proc. Biol. Sci. 281, 20141226-20141226

102 Lehmann, L. et al. (2006) Population demography and the evolution of helping behaviors. Evolution 60, 1137-1151

103 Powers, S.T. and Lehmann, L. (2013) The co-evolution of social institutions, demography, and large-scale human cooperation. Ecol Lett. 16, 1356-1364

104 McNally, L. et al. (2017) Killing by Type VI secretion drives genetic phase separation and correlates with increased cooperation. Nat. Commun. 8, 14371

105 Bourke, A.F.G. (2011) Principles of Social Evolution, OUP Oxford. 